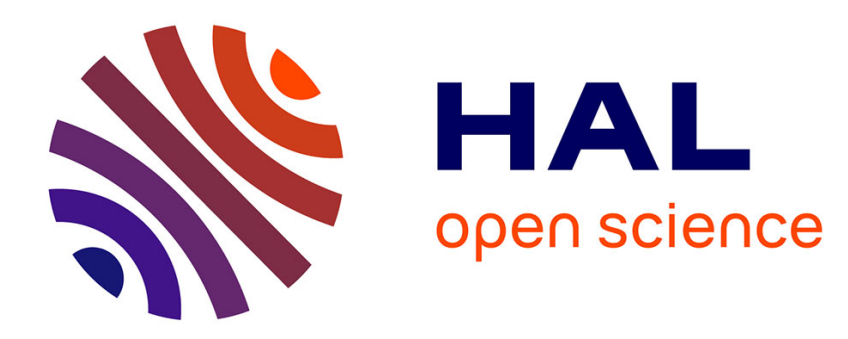

\title{
Ecosystem Viable Yields
}

Michel de Lara, Eladio Ocana Anaya, Ricardo Oliveros-Ramos, Jorge Tam

\section{To cite this version:}

Michel de Lara, Eladio Ocana Anaya, Ricardo Oliveros-Ramos, Jorge Tam. Ecosystem Viable Yields. 2011. hal-00587663v2

\section{HAL Id: hal-00587663 https://hal.science/hal-00587663v2}

Preprint submitted on 30 Nov 2011

HAL is a multi-disciplinary open access archive for the deposit and dissemination of scientific research documents, whether they are published or not. The documents may come from teaching and research institutions in France or abroad, or from public or private research centers.
L'archive ouverte pluridisciplinaire HAL, est destinée au dépôt et à la diffusion de documents scientifiques de niveau recherche, publiés ou non, émanant des établissements d'enseignement et de recherche français ou étrangers, des laboratoires publics ou privés. 


\title{
Ecosystem Viable Yields
}

\author{
Michel De Lara* Eladio OcaÑA ${ }^{\dagger} \quad$ Ricardo Oliveros-Ramos ${ }^{\ddagger}$ Jorge TAm ${ }^{\ddagger}$
}

November 30, 2011

\begin{abstract}
The World Summit on Sustainable Development (Johannesburg, 2002) encouraged the application of the ecosystem approach by 2010. However, at the same Summit, the signatory States undertook to restore and exploit their stocks at maximum sustainable yield (MSY), a concept and practice without ecosystemic dimension, since MSY is computed species by species, on the basis of a monospecific model. Acknowledging this gap, we propose a definition of "ecosystem viable yields" (EVY) as yields compatible i) with guaranteed biological safety levels for all time and ii) with an ecosystem dynamics. To the difference of MSY, this notion is not based on equilibrium, but on viability theory, which offers advantages for robustness. For a generic class of multispecies models with harvesting, we provide explicit expressions for the EVY. We apply our approach to the anchovy-hake couple in the Peruvian upwelling ecosystem.
\end{abstract}

Key words: control theory; state constraints; viability; yields; ecosystem management; Peruvian upwelling ecosystem.

\section{Introduction}

Following the World Summit on Sustainable Development (Johannesburg, 2002), the signatory States undertook to restore and exploit their stocks at maximum sustainable yield (MSY, see

\footnotetext{
*Université Paris-Est, CERMICS, 6-8 avenue Blaise Pascal, 77455 Marne la Vallée Cedex 2, France. Corresponding author: delara@cermics.enpc.fr, fax +33164153586

${ }^{\dagger}$ IMCA-FC, Universidad Nacional de Ingeniería, Calle los Biólogos 245, Lima 12-Perú. eocana@imca.edu.pe, fax $+511349-9838$

${ }^{\ddagger}$ IMARPE, Instituto del Mar del Perú, Centro de Investigaciones en Modelado Oceanográfico y Biológico Pesquero (CIMOBP), Apartado 22, Callao-Perú. jtam@imarpe.gob.pe, roliveros@imarpe.gob.pe, fax +5114535053
} 
(Clark, 1990)). Though being criticized for decades, MSY remains a reference. Criticisms of MSY, like (Larkin, 1977), point out that MSY relies upon a single variable stock description (the species biomass), without age structure nor interactions with other species; what is more, computations are made at equilibrium. In fisheries, one of the more elaborate method of fixing quotas, the ICES (International Council for the Exploration of the Sea) precautionary approach (ICES, 2004), does not assume equilibrium (it projects abundances one year ahead) and assumes age structure; it remains however based on a monospecific dynamical model. Thus, in fisheries, yields are usually defined species by species.

On the other hand, more and more emphasis is put on multispecies models (Hollowed, Bax, Beamish, Collie, Fogarty, Livingston, Pope, and Rice, 2000) and on ecosystem management. For instance, the World Summit on Sustainable Development encouraged the application of the ecosystem approach by 2010. Also, sustainability is a major goal of international agreements and guidelines to fisheries management (FAO, 1999; ICES, 2004).

Our interest is in providing conceptual insight as what could be sustainable yields for ecosystems. In this, we follow the vein of (Katz, Zabel, Harvey, Good, and Levin, 2003) which introduces the concept of Ecologically Sustainable Yield (ESY), or of (Chapel, Deffuant, Martin, and Mullon, 2008) which defines yield policies in a viability approach. A general discussion on the ecosystem approach to fisheries may be found in (Garcia, Zerbi, Aliaume, Chi, and Lasserre, 2003).

Our emphasis is on providing formal definition and practical methods to design and compute such yields. For this purpose, our approach is not based on equilibrium calculus, nor on intertemporal discounted utility maximization but on the so-called viability theory, as follows.

On the one hand, the ecosystem is described by a dynamical model controlled by harvesting. On the other hand, building upon (Béné, Doyen, and Gabay, 2001), constraints are imposed: catches are expected to be above given production minimal levels, and biomasses above safety biological minimal levels. Sustainability is here defined as the property that such constraints can be maintained for all time by appropriate harvesting strategies.

Such problems of dynamic control under constraints refer to viability (Aubin, 1991) or invariance (Clarke, Ledayev, Stern, and Wolenski, 1995) frameworks, as well as to reachability of target sets or tubes for nonlinear discrete time dynamics in (Bertsekas and Rhodes, 1971).

We consider sustainable management issues formulated within such framework as in (Béné, 
Doyen, and Gabay, 2001; Béné and Doyen, 2003; Eisenack, Sheffran, and Kropp, 2006; Mullon, Cury, and Shannon, 2004; Rapaport, Terreaux, and Doyen, 2006; De Lara, Doyen, Guilbaud, and Rochet, 2007; De Lara and Doyen, 2008; Chapel, Deffuant, Martin, and Mullon, 2008).

A viable state is an initial condition for the ecosystem dynamical system such that appropriate harvesting rules may drive the system on a sustainable path by maintaining catches and biomasses above their respective production and biological minimal levels. We provide a way to characterize production minimal levels (yields) such that the present initial conditions are a viable state. These yields are sustainable in the sense that they can be indefinitely guaranteed, while making possible that the ecosystem remains in an ecologically viable zone; we coin them ecosystem viable yields $(\mathrm{EVY})$.

Thus, the EVY can be seen as an extension of the MSY concept in two directions: 1) from equilibrium to viability (more robust); 2) from monospecies to multispecies models. The second claim is obvious because, as we have recalled at the beginning, the MSY relies upon a single variable stock description (the species biomass), without interactions with other species. As for the first, recall that the MSY is the largest constant yield that can be taken from a single species stock over an indefinite period. By contrast, EVY are guaranteed yields, but they are not necessarily the annual catches. Indeed, it is by an adaptive catch policy (depending on the states of the stocks) that we shall be able to display yields indefinitely above the EVY. This is why we say that EVY are guaranteed yields, in the sense that catches cannot fall below the EVY. Viability can be seen as a robust extension of equilibrium: yields are not supposed to be sustained by applying fixed stationary catches, but are minimal levels which can be guaranteed by means of adaptive catch policies.

The paper is organized as follows. In Section 2, we introduce generic harvested nonlinear ecosystem models, and we present how preservation and production constraints are modelled. Thanks to an explicit description of viable states, we are able to characterize sustainable yields. These latter are not defined species by species, but depend on the whole ecosystem dynamics and on all biological minimal levels. In Section 3, an illustration in ecosystem management and numerical applications are given for the hake-anchovy couple in the Peruvian upwelling ecosystem between the years 1971 and 1981. We conclude in Section 4 with possible extensions of the notion of ecosystem viable yields, on the one hand, to more general ecosystem models and, on the other hand, to 
bio-economic models so as to incorporate some economic considerations. We also discuss the limits of the EVY concept. In the appendix, Section A is devoted to recalls on discrete-time viability and its possible use for sustainable management, while Section B contains the mathematical proofs.

\section{Ecosystem viable yields}

After a brief recall on the notion of maximum sustainable yield (for monospecific models), we introduce a class of generic harvested nonlinear ecosystem models, then present how to define maximum sustainable yields for this class. Next, we provide an explicit description of viable states, for which production and biological constraints can be guaranteed for all times under appropriate management. This makes possible to define ecosystem viable yields, compatible with biological and conservation constraints. We end up discussing relations between ecosystem viable yields and maximum sustainable yields.

\subsection{A brief recall on maximum sustainable yield}

We briefly sketch the principles leading to the notion of maximum sustainable yield (MSY) (see (Clark, 1990) in continuous time and (De Lara and Doyen, 2008) in discrete time).

Consider a single population described by its total biomass $B(t)$ at time $t$. Suppose that the time evolution of the biomass is given by a dynamical equation, a differential equation $\dot{B}(t)=$ $\mathrm{Biol}_{c}(B(t))$ in continuous time or a difference equation $B(t+1)=\mathrm{Biol}_{d}(B(t))$ in discrete time. From this, build a Schaefer model (Schaefer, 1954) by substracting a catch term $h(t)$, giving $\dot{B}(t)=$ $\mathrm{Biol}_{c}(B(t))-h(t)$ or $B(t+1)=\mathrm{Biol}_{d}(B(t)-h(t))$. In general, to each biomass level $B_{\mathrm{E}}$ (below the carrying capacity) corresponds a catch level $h_{\mathrm{E}}=\operatorname{Sust}\left(B_{\mathrm{E}}\right)$ for which the biomass $B_{\mathrm{E}}$ is at equilibrium, solution of Biol ${ }_{c}\left(B_{\mathrm{E}}\right)-h_{\mathrm{E}}=0$ or Biol $_{d}\left(B_{\mathrm{E}}-h_{\mathrm{E}}\right)=B_{\mathrm{E}}$. The maximum sustainable yield is the largest of such equilibrium catches: $\mathrm{MSY}=\max _{B_{\mathrm{E}}} \operatorname{Sust}\left(B_{\mathrm{E}}\right)$.

\subsection{Ecosystem biomass dynamical model}

For simplicity, we consider a model with two species, but it can be easiliy extended to $N$ species in interaction. Each species is described by its biomass: the two-dimensional state vector $(y, z)$ represents the biomasses of both species. The two-dimensional control $(v, w)$ comprises the harvesting 
effort for each species, respectively. The catches are thus $v y$ and $w z$ (measured in biomass). ${ }^{1}$ The discrete-time control system we consider is

$$
\left\{\begin{array}{l}
y(t+1)=y(t) R_{y}(y(t), z(t), v(t)), \\
z(t+1)=z(t) R_{z}(y(t), z(t), w(t)),
\end{array}\right.
$$

where $t$ stand for time (typically, periods are years), and where $R_{y}: \mathbb{R}^{3} \rightarrow \mathbb{R}$ and $R_{z}: \mathbb{R}^{3} \rightarrow \mathbb{R}$ are two functions representing growth factors (the growth rates being $R_{y}-1$ and $R_{z}-1$ ). This model is generic in that no explicit or analytic assumptions are made on how the growth factors $R_{y}$ and $R_{z}$ indeed depend upon both biomasses $(y, z)$.

In the above model, each species is harvested by a specific device: one species, one harvesting effort. This covers the multioutput settings case (e.g. several species in trophic interactions and targeted by the same fishing gear). Indeed, for this it suffices to state that both efforts are identical: $v(t)=w(t)$ for all $t=t_{0}, t_{0}+1, \ldots$

\subsection{Preservation and production sustainability}

We now propose to define sustainability as the ability to respect preservation and production minimal levels for all times, building upon the original approach of (Béné, Doyen, and Gabay, 2001). Let us be given

- on the one hand, minimal biomass levels $B_{y}^{b} \geq 0, B_{z}^{b} \geq 0$, one for each species,

- on the other hand, minimal catch levels $C_{y}^{b} \geq 0, C_{z}^{b} \geq 0$, one for each species.

A couple $\left(y_{0}, z_{0}\right)$ of initial biomasses is said to be a viable state if there exist appropriate harvesting efforts (controls) $(v(t), w(t)), t=t_{0}, t_{0}+1, \ldots$ such that the state path $(y(t), z(t)), t=$ $t_{0}, t_{0}+1, \ldots$ starting from $\left(y\left(t_{0}\right), z\left(t_{0}\right)\right)=\left(y_{0}, z_{0}\right)$ satisfies the following goals:

- preservation (minimal biomass levels)

$$
\text { biomasses: } y(t) \geq B_{y}^{b}, \quad z(t) \geq B_{z}^{b}, \quad \forall t=t_{0}, t_{0}+1, \ldots
$$

\footnotetext{
${ }^{1}$ In fact, any expression of the form $c(y, v)$, instead of $v y$, would fit for the catches in the following Proposition 2 as soon as $v \mapsto c(y, v)$ is strictly increasing and goes from 0 to $+\infty$ when $v$ goes from 0 to $+\infty$. The same holds for $d(z, w)$ instead of $w z$.
} 
- and production requirements (minimal catch levels)

$$
\text { catches: } v(t) y(t) \geq C_{y}^{b}, \quad w(t) z(t) \geq C_{z}^{b}, \quad \forall t=t_{0}, t_{0}+1, \ldots
$$

The set of all viable states is called the viability kernel (Aubin, 1991). Characterizing viable states makes it possible to test whether or not minimal biomasses and catches can be guaranteed for all time.

Here, by guaranteed, we mean that the yields have to indefinitely above the EVY, as reflected in the inequalities (2) and (3). We insist on the fact that, in the above definition of viable states, we say "there exist appropriate harvesting efforts (controls)": that is, to guarantee the EVY, we need to resort to adaptive catch policies (depending on the states of the stocks as in Corollary 6 in the Appendix). Viability can be seen as a robust extension of equilibrium: yields are not supposed to be sustained by applying fixed stationary catches, but are minimal levels which can be guaranteed by means of adaptive catch policies.

Notice that, in the multioutput settings case, we need to add the constraint $v(t)=w(t)$ for all $t=t_{0}, t_{0}+1, \ldots$. Therefore, with an additional constraint, the set of viable states in the multioutput settings case will be smaller than the one considered above.

The following definition summarizes useful and natural properties required for the growth factors in the ecosystem model.

Definition 1 We say that growth factors $R_{y}$ and $R_{z}$ in the ecosystem model (1) are nice if the function $R_{y}: \mathbb{R}^{3} \rightarrow \mathbb{R}$ is continuously decreasing ${ }^{2}$ in the harvesting effort $v$ and satisfies $\lim _{v \rightarrow+\infty} R_{y}(y, z, v) \leq 0$, and if $R_{z}: \mathbb{R}^{3} \rightarrow \mathbb{R}$ is continuously decreasing in the harvesting effort $w$, and satisfies $\lim _{w \rightarrow+\infty} R_{z}(y, z, w) \leq 0$.

The following Proposition 2 gives an explicit description of the viable states, under some conditions on the minimal levels. Its proof is given in $\S$ B.1 in the Appendix.

The Proposition 2 may easily be extended to $N$ species in interaction as long as each species is harvested by a specific device: one species, one harvesting effort. However, it is not valid in the multioutput settings case. Indeed, it is crucial to have two distinct controls $v(t)$ and $w(t)$ for the

\footnotetext{
${ }^{2}$ In all that follows, a mapping $\varphi: \mathbb{R} \rightarrow \mathbb{R}$ is said to be increasing if $x \geq x^{\prime} \Rightarrow \varphi(x) \geq \varphi\left(x^{\prime}\right)$. The reverse holds for decreasing. Thus, with this definition, a constant mapping is both increasing and decreasing.
} 
proof. Assuming that $v(t)=w(t)$ for all $t=t_{0}, t_{0}+1, \ldots$ would require other types of calculations for the viability kernel. This is out of the scope of this paper.

Proposition 2 Assume that the growth factors in the ecosystem model (1) are nice. If the biomass minimal levels $B_{y}^{b}, B_{z}^{b}$, and the catch minimal levels $C_{y}^{b}, C_{z}^{b}$ are such that the following growth factors values are greater than one

$$
R_{y}\left(B_{y}^{b}, B_{z}^{b}, \frac{C_{y}^{b}}{B_{y}^{b}}\right) \geq 1 \text { and } R_{z}\left(B_{y}^{b}, B_{z}^{b}, \frac{C_{z}^{b}}{B_{z}^{b}}\right) \geq 1,
$$

then the viable states are all the couples $(y, z)$ of biomasses such that

$$
y \geq B_{y}^{b}, \quad z \geq B_{z}^{b}, \quad y R_{y}\left(y, z, \frac{C_{y}^{b}}{y}\right) \geq B_{y}^{b}, \quad z R_{z}\left(y, z, \frac{C_{z}^{b}}{z}\right) \geq B_{z}^{b} .
$$

Let us comment the assumptions of Proposition 2. That the growth factors are decreasing with respect to the harvesting effort is a natural assumption. Conditions (4) mean that, at the point $\left(B_{y}^{b}, B_{z}^{b}\right)$ and applying efforts $u^{b}=\frac{C_{y}^{b}}{B_{y}^{b}}, v^{b}=\frac{C_{z}^{b}}{B_{z}^{b}}$, the growth factors are greater than one, hence both populations grow; hence, it could be thought that computing viable states is useless since everything looks fine. However, if all is fine at the point $\left(B_{y}^{b}, B_{z}^{b}\right)$, it is not obvious that this also goes for a larger domain. Indeed, the ecosystem dynamics given by (1) has no monotonocity properties that would allow to extend a result valid for a point to a whole domain. What is more, if continuous-time viability results mostly relies upon assumptions at the frontier of the constraints set, this is no longer true for discrete-time viability.

\subsection{Ecosystem viable yields}

Considering that minimal biomass conservation levels are given first (for prominent biological issues), we shall now examine conditions for the existence of minimal catch levels

First, we define (when they exist) the ecosystem viable yields.

Definition 3 Let biomass conservation minimal levels $B_{y}^{b} \geq 0, B_{z}^{b} \geq 0$ be given. Suppose that the growth factors in the ecosystem model (1) are nice, and that they take values greater than one in the absence of harvesting, namely:

$$
R_{y}\left(B_{y}^{b}, B_{z}^{b}, 0\right) \geq 1 \text { and } R_{z}\left(B_{y}^{b}, B_{z}^{b}, 0\right) \geq 1
$$


Define equilibrium catches as the largest nonnegative ${ }^{3}$ catches $C_{y}^{b, \star}, C_{z}^{b, \star}$ such that

$$
R_{y}\left(B_{y}^{b}, B_{z}^{b}, \frac{C_{y}^{b, \star}}{B_{y}^{b}}\right)=1 \text { and } R_{z}\left(B_{y}^{b}, B_{z}^{b}, \frac{C_{z}^{b, \star}}{B_{z}^{b}}\right)=1
$$

For a couple $\left(y_{0}, z_{0}\right)$ of biomasses, define (when they exist) the ecosystem viable yields (EVY) $C_{y}^{b, \star}\left(y_{0}, z_{0}\right)$ and $C_{z}^{b, \star}\left(y_{0}, z_{0}\right)$ by

$$
\left\{\begin{array}{l}
C_{y}^{b, \star}\left(y_{0}, z_{0}\right):=\max \left\{C_{y} \in\left[0, C_{y}^{b, \star}\right] \mid y_{0} R_{y}\left(y_{0}, z_{0}, \frac{C_{y}}{y_{0}}\right) \geq B_{y}^{b}\right\} \\
C_{z}^{b, \star}\left(y_{0}, z_{0}\right):=\max \left\{C_{z} \in\left[0, C_{z}^{b, \star}\right] \mid z_{0} R_{z}\left(y_{0}, z_{0}, \frac{C_{z}}{z_{0}}\right) \geq B_{z}^{b}\right\} .
\end{array}\right.
$$

The term ecosystem viable yields is justified by the following Proposition 4 .

Proposition 4 Assume that the growth factors in the ecosystem model (1) are nice. For a couple $\left(y_{0}, z_{0}\right)$ of biomasses above preservation minimal levels - that is, $y_{0} \geq B_{y}^{b}$ and $z_{0} \geq B_{z}^{b}-$ and satisfying

$$
y_{0} R_{y}\left(y_{0}, z_{0}, 0\right) \geq B_{y}^{b} \text { and } z_{0} R_{z}\left(y_{0}, z_{0}, 0\right) \geq B_{z}^{b}
$$

the ecosystem viable yields $C_{y}^{b, \star}\left(y_{0}, z_{0}\right)$ and $C_{z}^{b, \star}\left(y_{0}, z_{0}\right)$ in (8) are well defined.

What is more, consider catches $C_{y}^{b}$ and $C_{z}^{b}$ lower than these ecosystem viable yields, that is, $0 \leq C_{y}^{b} \leq C_{y}^{b, \star}\left(y_{0}, z_{0}\right)$ and $0 \leq C_{z}^{b} \leq C_{z}^{b, \star}\left(y_{0}, z_{0}\right)$. Then, starting from the initial biomasses $\left(y\left(t_{0}\right), z\left(t_{0}\right)\right)=\left(y_{0}, z_{0}\right)$, there exists appropriate harvesting paths which provide, for all time, at least the sustainable yields $C_{y}^{b}$ and $C_{z}^{b}$ and which guarantee that biomass conservation minimal levels $B_{y}^{b} \geq 0, B_{z}^{b} \geq 0$ are respected for all time.

From the practical point of view, the upper quantities $C_{y}^{b, \star}\left(y_{0}, z_{0}\right)$ and $C_{z}^{b, \star}\left(y_{0}, z_{0}\right)$ in (8) cannot be seen as catches targets, but rather as crisis limits. Indeed, the closer to them, the more vulnerable, since the initial point is close to the viability kernel boundary.

Notice that the yield $C_{y}^{b, \star}\left(y_{0}, z_{0}\right)$ depends, first, on both species biomasses $\left(y_{0}, z_{0}\right)$, second, on both conservation minimal levels $B_{y}^{b}$ and $B_{z}^{b}$, third, on the ecosystem model by the growth factor $R_{y}$; the same holds for $C_{z}^{b, \star}\left(y_{0}, z_{0}\right)$. Thus, these yields are designed jointly on the basis of the whole ecosystem model and of all the conservation minimal levels; this is why we coined them ecosystem viable yields.

\footnotetext{
${ }^{3}$ Such catches are nonnegative because the growth factors in the ecosystem model (1) are nice, hence continuously decreasing in the harvesting effort, and by (6).
} 
This observation may have practical consequences. Indeed, the catches guaranteed for one species depend not only on the biological minimal level of the same species, but on the other species. For instance, in the Peruvian upwelling ecosystem, it is customary to increase the biological minimal level of the anchovy before an El Niño event, but without explicitely considering to lower catches of other species. Our analysis stresses the point that minimal levels have to be designed globally to guarantee sustainability for the whole ecosystem.

\subsection{Ecosystem viable yields and maximum sustainable yields}

Now, we show how ecosystem viable yields are related to maximum sustainable yields.

An equilibrium of the ecosystem model (1) is a couple $\left(y_{\mathrm{E}}, z_{\mathrm{E}}\right)$ of biomasses (state) and a couple $\left(v_{\mathrm{E}}, w_{\mathrm{E}}\right)$ of harvesting efforts (control) satisfying

$$
\left\{\begin{array}{l}
y_{\mathrm{E}}=y_{\mathrm{E}} R_{y}\left(y_{\mathrm{E}}, z_{\mathrm{E}}, v_{\mathrm{E}}\right), \\
z_{\mathrm{E}}=z_{\mathrm{E}} R_{z}\left(y_{\mathrm{E}}, z_{\mathrm{E}}, w_{\mathrm{E}}\right) .
\end{array}\right.
$$

The maximum sustainable yields, $\mathrm{MSY}_{y}$ for species $y$ and $\mathrm{MSY}_{z}$ for species $z$, are given by

$$
\mathrm{MSY}_{y}:=\max _{v_{\mathrm{E}}, w_{\mathrm{E}}} v_{\mathrm{E}} y_{\mathrm{E}} \text { and } \mathrm{MSY}_{z}:=\max _{v_{\mathrm{E}}, w_{\mathrm{E}}} w_{\mathrm{E}} z_{\mathrm{E}} \cdot
$$

They must be jointly defined because the ecosystem equilibrium equations (10) couple all variables.

Say that the maximum sustainable yields $\mathrm{MSY}_{y}$ and $\mathrm{MSY}_{z}$ are viable maximum sustainable yields if the corresponding biomasses equilibrium values $y_{\mathrm{E}}$ and $z_{\mathrm{E}}$ are such that $y_{\mathrm{E}} \geq B_{y}^{b}$ and $z_{\mathrm{E}} \geq B_{z}^{b}$. In this case, $\mathrm{MSY}_{y}$ and $\mathrm{MSY}_{z}$ are ecosystem viable yields for the couple $\left(y_{\mathrm{E}}, z_{\mathrm{E}}\right)$ of initial biomasses: indeed, the stationary harvest strategy $v(t)=v_{\mathrm{E}}$ and $w(t)=w_{\mathrm{E}}$ drives the ecosystem model (1) at equilibrium $\left(y_{\mathrm{E}}, z_{\mathrm{E}}\right)$ which satisfies the conservation minimal levels $y_{\mathrm{E}} \geq B_{y}^{b}$ and $z_{\mathrm{E}} \geq B_{z}^{b}$.

Notice that the maximum sustainable yields $\mathrm{MSY}_{y}$ and $\mathrm{MSY}_{z}$ are defined independently of the initial biomasses, whereas the ecosystem viable yields (EVY) $C_{y}^{b, \star}\left(y_{0}, z_{0}\right)$ and $C_{z}^{b, \star}\left(y_{0}, z_{0}\right)$ explicitely depend upon them.

\section{Numerical application to the hake-anchovy couple in the Peru- vian upwelling ecosystem (1971-1981)}

We provide a viability analysis of the hake-anchovy Peruvian fisheries between the years 1971 and 1981. For this, we shall consider a discrete-time Lotka-Volterra model for the couple anchovy (prey 
$y$ ) and hake (predator $z$ ), then provide an explicit description of viable states.

We warn the reader that our emphasis is not on developing a "knowledge" biological model to "faithfully" describe the complexity of the Peruvian upwelling ecosystem. This formidable task is out of our competencies, and is not necessary for our analysis. Indeed, our approach makes use, not of "knowledge" models, but of "action" models; these are small, compact, models which capture essentials features of the system in what concern decision-making. In our case, we needed a compact model able to put in consistency biomass and catches yearly data between the years 1971 and 1981. We chose a discrete-time Lotka-Volterra model, despite well-known criticisms as candidate for a "knowledge" biological model (Hall, 1988; Murray, 2002), but for its compactness qualities and for the reasonable fit (see Figure 1).

\subsection{Viable states and ecosystem sustainable yields for a Lotka-Volterra system}

Consider the following discrete-time Lotka-Volterra system of equations with density-dependence in the prey

$$
\left\{\begin{array}{l}
y(t+1)=R y(t)-\frac{R}{\kappa} y^{2}(t)-\alpha y(t) z(t)-v(t) y(t) \\
z(t+1)=L z(t)+\beta y(t) z(t)-w(t) z(t)
\end{array}\right.
$$

where $R>1,0<L<1, \alpha>0, \beta>0$ and $\kappa=\frac{R}{R-1} K$, with $K>0$ the carrying capacity for prey. In the dynamics (1), we identify $R_{y}(y, z, v)=R-\frac{R}{\kappa} y-\alpha z-v$ and $R_{z}(y, w)=L+\beta y-w$.

By Proposition 4 , we obtain that, for any initial point $\left(y_{0}, z_{0}\right)$ such that

$$
y_{0} \geq B_{y}^{b}, \quad z_{0} \geq B_{z}^{b}, \quad y_{0}\left(R-\frac{R}{\kappa} y_{0}-\alpha z_{0}\right) \geq B_{y}^{b},
$$

the ecosystem sustainable yields are given by

$$
\left\{\begin{array}{l}
C_{y}^{b, \star}\left(y_{0}, z_{0}\right)=\min \left\{B_{y}^{b}\left(R-\frac{R}{\kappa} B_{y}^{b}-\alpha B_{z}^{b}\right)-B_{y}^{b}, y_{0}\left(R-\frac{R}{\kappa} y_{0}-\alpha z_{0}\right)-B_{y}^{b}\right\} \\
C_{z}^{b, \star}\left(y_{0}, z_{0}\right)=B_{z}^{b}\left(L+\beta B_{y}^{b}-1\right)
\end{array}\right.
$$

In other words, if viably managed, the ecosystem could produce at least $C_{y}^{b, \star}\left(y_{0}, z_{0}\right)$ and $C_{z}^{b, \star}\left(y_{0}, z_{0}\right)$, while respecting biological minimal levels $B_{y}^{b}$ and $B_{z}^{b}$. 


\subsection{A viability analysis of the hake-anchovy Peruvian fisheries between the years 1971 and 1981}

The Peruvian upwelling ecosystem is extremely productive and dominated by anchovy (Engraulis ringens) dynamics. It is well known that anchovy fisheries are very sensitive to environmental variability (Checkley, Alheit, Ooseki, and Roy, 2009; Sun, Chiang, Liu, and Chang, 2001), and the Peruvian anchovy is subject to environmental perturbations such as El Niño Southern Oscillation (ENSO) variability (Bertrand, Segura, Gutiérrez, and Vásquez, 2004)). However, for this simple predator-prey model using hake and anchovy, we have assumed that no uncertainties affect the ecosystem dynamics. Indeed, we feel that we have to go step by step in introducing the EVY concept, first focusing on the deterministic case. Thus, the period between the years 1971 and 1981 is suitable for this first version of the model, due to the absence of strong El Niño events in the middle of the period. Furthermore, the long-term dynamics of the Peruvian upwelling ecosystem is dominated by shifts between alternating anchovy and sardine regimes that restructure the entire ecosystem (Alheit and Niquen, 2004). The period from 1970 to 1985 was characterized by positive temperature anomalies and low anchovy abundances, after the anchovy collapse in 1971 (Alheit and Niquen, 2004), so the competition between the fishery and hake was reduced due to low anchovy catches and anchovy mortality due to hake predation increase. Particularly, the changes in the ecosystem after 1971 led to an increase of five times, in average, in the predation rates of hake over anchovy between 1971 to 1980, with a peak in 1977 (Pauly and Palomares, 1989).

Between the years 1971 and 1981, we have 11 couples of biomasses, and the same for catches. The 5 parameters of the Lotka-Volterra model are estimated minimizing a weighted residual squares sum function using a conjugate gradient method, with central derivatives. Estimated parameters and comparisons of observed and simulated biomasses are shown in Figure 1.

We consider values of $B_{y}^{b}=7,000,000 \mathrm{t}$ and $B_{z}^{b}=200,000 \mathrm{t}$ for minimal biomass levels (IMARPE, 2000, 2004). Conditions (13) are satisfied and the expressions (14) give the ecosystem viable yields (EVY)

$$
C_{y}^{b, \star}\left(y_{0}, z_{0}\right)=5,399,000 \mathrm{t} \text { and } C_{z}^{b, \star}\left(y_{0}, z_{0}\right)=56,800 \mathrm{t}
$$

In other words, such yields were theoreticaly susceptible to be guaranteed in a sustainable way starting from year 1971. In reality, the catches of year 1971 were very high and the biomasses trajectories were well below the biological minimal levels for fourteen years. 


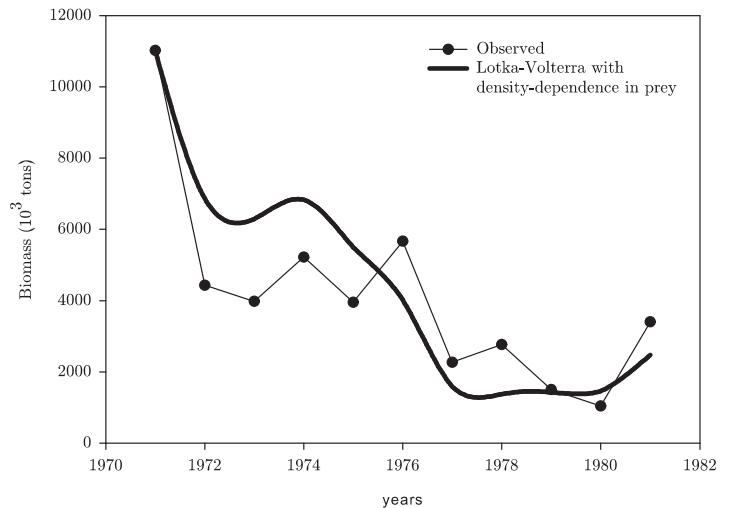

(a) Anchovy

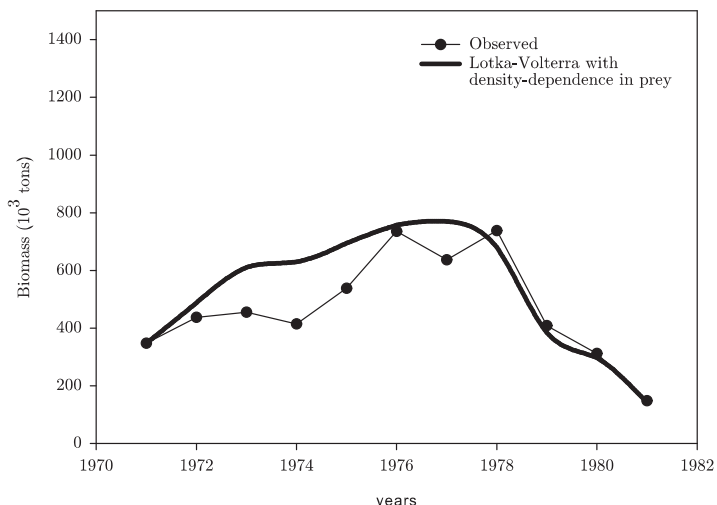

(b) Hake

Figure 1: Comparison of observed and simulated biomasses of anchovy and hake using a LotkaVolterra model with density-dependence in the prey (1971-1981). Model parameters are $R=$ 2.25 year $^{-1}, L=0.945$ year $^{-1}, \kappa=67,113 \times 10^{3} \mathrm{t}\left(K=37,285 \times 10^{3} \mathrm{t}\right), \alpha=1.220 \times 10^{-6} \mathrm{t}^{-1}$, $\beta=4.845 \times 10^{-8} \mathrm{t}^{-1}$.

The 4,250,000 t anchovy quota and the 55,000 t hake quota, respectively, established for the year 2006 (PRODUCE, 2005), or the 5,000,000 $\mathrm{t}$ anchovy quota and the 35,000 $\mathrm{t}$ hake quota, respectively, established for the year 2007 (PRODUCE, 2006) are rather close to the EVY $C_{y}^{b, \star}\left(y_{0}, z_{0}\right)=5,399,000 \mathrm{t}$ and $C_{z}^{b, \star}\left(y_{0}, z_{0}\right)=56,800$ t. Thus, our approach provides reasonable figures. ${ }^{4}$

\section{Conclusion}

We have defined the notion of sustainable yields for ecosystem, and provided ways to compute them by means of a viability analysis of generic ecosystem models with harvesting. Our analysis stresses the point that yields should certainly be designed globally, and not species by species as in the current practice, to guarantee sustainability for the whole ecosystem. Our results have then been applied to a Lotka-Volterra model using the anchovy-hake couple in the Peruvian upwelling

\footnotetext{
${ }^{4}$ At this stage, we do not claim that the figures may be proposed as yields for the present management of hakeanchovy Peruvian fisheries. Indeed, our computations of EVY rely upon a dynamical model adjusted for some thirty years ago. To propose EVY, we should first dispose of a dynamical model adapted to the current situation, because it ought to reflect the new ecosystem functioning and the depleted state of stocks (Ballón, Wosnitza-Mendo, Guevara-Carrasco, and Bertrand, 2008). This is beyond the scope of this paper.
} 
ecosystem. Despite simplicity ${ }^{5}$ of the models considered, our approach has provided reasonable figures and new insights: it may be a mean of designing sustainable yields from an ecosystem point of view.

We now discuss the limits of the EVY concept, as presented in this paper: application to biomass ecosystem models without age or spatial structure, no economic consideration, no uncertainties. We stress that EVY is a flexible concept, and we hint at possible extensions to incorporate the missing dimensions listed above.

The framework we propose is not restricted to two populations, each described by its global biomass, but it may be adapted to several species, each described by a vector of abundances at age, or by vectors of abundances at age for each patch in a spatial model, etc. Suppose that the time evolution is given by a dynamical equation reflecting ecosystemic interactions and driven by efforts or by catches. Suppose that minimal safety levels (reference points) are fixed for biological indicators like spawning stock biomass, abundances at specific ages, etc. (such reference points for biological indicators like spawning stock biomass are generally given by international bodies like the ICES, or nationally). Ecosystem viable yields are minimal harvests for each species which can be guaranteed for all times while respecting the above minimal safety levels for biological indicators for all times too.

It is often objected with reason that the MSY concept is developed without any economic consideration. As presented here, the EVY suffers the same criticism. However, the EVY concept is flexible enough to incorporate some economic considerations. For instance, upper bounds for fishing costs may be incorporated as constraints to be satisfied for all time, aside with minimal biomass levels. In this sense, EVY will be guaranteed yields compatible with biological and economic restrictions.

As presented here, the EVY framework supposes that no uncertainties affect the ecosystem dynamics. Though we have the tools to tackle such an important issue (see stochastic viability in (De Lara and Doyen, 2008; De Lara and Martinet, 2009; Doyen and De Lara, 2010)), we feel that we have to go step by step. This paper introduces the EVY concept in the deterministic case,

\footnotetext{
${ }^{5}$ In addition to hake, there are other important predators of anchovy in the Peruvian upwelling ecosystem, such as mackerel and horse mackerel, seabirds and pinnipeds, which were not considered. Also, anchovy has been an important prey of hake, but other prey species have been found in the opportunistic diet of hake (Tam, Purca, Duarte, Blaskovic, and Espinoza, 2006)
} 
providing an extension of the MSY concept in two directions: from equilibrium to viability (more robust), from monospecies to multispecies models. The extension to the uncertain case is currently under investigation.

Thus, control and viability theory methods have allowed us to introduce ecosystem considerations, such as multispecies and multiobjectives, and have contributed to integrate the long term dynamics, which is generally not considered in conventional fishery management.

Acknowledgments. This paper was prepared within the MIFIMA (Mathematics, Informatics and Fisheries Management) international research network; we thank CNRS, INRIA and the French Ministry of Foreign Affairs for their funding and support through the regional cooperation program STIC-AmSud. We thank the staff of the Peruvian Marine Research Institute (IMARPE), especially Erich Diaz and Nathaly Vargas for discussions on anchovy and hake fisheries. We thank Sophie Bertrand and Arnaud Bertrand from IRD at IMARPE for their insightful comments. We also thank Yboon Garcia (IMCA-Peru and CMM-Chile) for a discussion on the ecosystem model case. We are particularly indebted to the reviewer who, by his/her comments and questions, helped us improve the presentation.

\section{A Discrete-time viability}

Let us consider a nonlinear control system described in discrete-time by the dynamic equation

$$
\left\{\begin{array}{l}
x(t+1)=f(x(t), u(t)) \text { for all } t \in \mathbb{N}, \\
x(0)=x_{0} \text { given, }
\end{array}\right.
$$

where the state variable $x(t)$ belongs to the finite dimensional state space $\mathbb{X}=\mathbb{R}^{n_{\mathbb{X}}}$, the control variable $u(t)$ is an element of the control set $\mathbb{U}=\mathbb{R}^{n_{\mathbb{U}}}$ while the dynamics $f$ maps $\mathbb{X} \times \mathbb{U}$ into $\mathbb{X}$.

A controller or a decision maker describes "acceptable configurations of the system" through a set $\mathbb{D} \subset \mathbb{X} \times \mathbb{U}$ termed the acceptable set

$$
(x(t), u(t)) \in \mathbb{D} \text { for all } t \in \mathbb{N},
$$

where $\mathbb{D}$ includes both system states and controls constraints. 
The state constraints set $\mathbb{V}^{0}$ associated with $\mathbb{D}$ is obtained by projecting the acceptable set $\mathbb{D}$ onto the state space $\mathbb{X}$ :

$$
\mathbb{V}^{0}:=\operatorname{Proj}_{\mathbb{X}}(\mathbb{D})=\{x \in \mathbb{X} \mid \exists u \in \mathbb{U},(x, u) \in \mathbb{D}\}
$$

Viability is defined as the ability to choose, at each time step $t \in \mathbb{N}$, a control $u(t) \in \mathbb{U}$ such that the system configuration remains acceptable. More precisely, viability occurs when the following set of initial states is not empty:

$$
\mathbb{V}(f, \mathbb{D}):=\left\{\begin{array}{l|l}
x_{0} \in \mathbb{X} & \begin{array}{l}
\exists(u(0), u(1), \ldots) \text { and }(x(0), x(1), \ldots) \\
\text { satisfying (16) and (17) }
\end{array}
\end{array}\right\} .
$$

The set $\mathbb{V}(f, \mathbb{D})$ is called the viability kernel (Aubin, 1991) associated with the dynamics $f$ and the acceptable set $\mathbb{D}$. By definition, we have $\mathbb{V}(f, \mathbb{D}) \subset \mathbb{V}^{0}=\operatorname{Proj}_{\mathbb{X}}(\mathbb{D})$ but, in general, the inclusion is strict. For a decision maker or control designer, knowing the viability kernel has practical interest since it describes the initial states for which controls can be found that maintain the system in an acceptable configuration forever. However, computing this kernel is not an easy task in general.

We now focus on some tools to achieve viability. A subset $\mathbb{V}$ is said to be weakly invariant for the dynamics $f$ in the acceptable set $\mathbb{D}$, or a viability domain of $f$ in $\mathbb{D}$, if

$$
\forall x \in \mathbb{V}, \quad \exists u \in \mathbb{U}, \quad(x, u) \in \mathbb{D} \text { and } f(x, u) \in \mathbb{V} .
$$

That is, if one starts from $\mathbb{V}$, an acceptable control may transfer the state in $\mathbb{V}$. Moreover, according to viability theory (Aubin, 1991), the viability kernel $\mathbb{V}(f, \mathbb{D})$ turns out to be the union of all viability domains, or also the largest viability domain:

$$
\mathbb{V}(f, \mathbb{D})=\bigcup\left\{\mathbb{V}, \mathbb{V} \subset \mathbb{V}^{0}, \mathbb{V} \text { viability domain for } f \text { in } \mathbb{D}\right\} .
$$

Viable controls are those controls $u \in \mathbb{U}$ such that $(x, u) \in \mathbb{D}$ and $f(x, u) \in \mathbb{V}(f, \mathbb{D})$.

A major interest of such a property lies in the fact that any viability domain for the dynamics $f$ in the acceptable set $\mathbb{D}$ provides a lower approximation of the viability kernel. An upper approximation $\mathbb{V}_{k}$ of the viability kernel is given by the so called viability kernel until time $k$ associated with $f$ in $\mathbb{D}$ :

$$
\mathbb{V}_{k}:=\left\{\begin{array}{l|l}
x_{0} \in \mathbb{X} & \begin{array}{l}
\exists(u(0), u(1), \ldots, u(k)) \text { and }(x(0), x(1), \ldots, x(k)) \\
\text { satisfying (16) for } t=0, \ldots, k-1 \\
\text { and (17) for } t=0, \ldots, k
\end{array}
\end{array}\right\} .
$$


We have

$$
\mathbb{V}(f, \mathbb{D}) \subset \mathbb{V}_{k+1} \subset \mathbb{V}_{k} \subset \mathbb{V}_{0}=\mathbb{V}^{0} \text { for all } k \in \mathbb{N}
$$

It may be seen by induction that the decreasing sequence of viability kernels until time $k$ satisfies

$$
\mathbb{V}_{0}=\mathbb{V}^{0} \text { and } \mathbb{V}_{k+1}=\left\{x \in \mathbb{V}_{k} \mid \exists u \in \mathbb{U},(x, u) \in \mathbb{D} \text { and } f(x, u) \in \mathbb{V}_{k}\right\}
$$

By (23), such an algorithm provides approximation from above of the viability kernel as follows:

$$
\mathbb{V}(f, \mathbb{D}) \subset \bigcap_{k \in \mathbb{N}} \mathbb{V}_{k}=\lim _{k \rightarrow+\infty} \downarrow \mathbb{V}_{k}
$$

Conditions ensuring that equality holds may be found in (Saint-Pierre, 1994). Notice that, when the decreasing sequence $\left(\mathbb{V}_{k}\right)_{k \in \mathbb{N}}$ of viability kernels up to time $k$ is stationary, its limit is the viability kernel. Indeed, if $\mathbb{V}_{k}=\mathbb{V}_{k+1}$ for some $k$, then $\mathbb{V}_{k}$ is a viability domain by (24). Now, by (19), $\mathbb{V}(f, \mathbb{D})$ is the largest of viability domains. As a consequence, $\mathbb{V}_{k}=\mathbb{V}(f, \mathbb{D})$ since $\mathbb{V}(f, \mathbb{D}) \subset \mathbb{V}_{k}$ by (23). We shall use this property in the following Sect. B.

\section{B Viable control of generic nonlinear ecosystem models with har- vesting}

For a generic ecosystem model (1), we provide an explicit description of the viability kernel. Then, we shall specify the results for predator-prey systems, in particular for discrete-time Lotka-Volterra models.

The acceptable set $\mathbb{D}$ in (17) is defined by minimal biomass levels $B_{y}^{b} \geq 0, B_{z}^{b} \geq 0$ and minimal catch levels $C_{y}^{b} \geq 0, C_{z}^{b} \geq 0$ :

$$
\mathbb{D}=\left\{(y, z, v, w) \in \mathbb{R}^{4} \mid y \geq B_{y}^{b}, z \geq B_{z}^{b}, v y \geq C_{y}^{b}, w z \geq C_{z}^{b}\right\}
$$

\section{B.1 Expression of the viability kernel}

The following Proposition 5 gives an explicit description of the viability kernel, under some conditions on the minimal levels.

Proposition 5 Assume that the function $R_{y}: \mathbb{R}^{3} \rightarrow \mathbb{R}$ is continuously decreasing in the control $v$ and satisfies $\lim _{v \rightarrow+\infty} R_{y}(y, z, v) \leq 0$, and that $R_{z}: \mathbb{R}^{3} \rightarrow \mathbb{R}$ is continuously decreasing in the 
control variable $w$, and satisfies $\lim _{w \rightarrow+\infty} R_{z}(y, z, w) \leq 0$. If the minimal levels in (26) are such that the following growth factors are greater than one

$$
R_{y}\left(B_{y}^{b}, B_{z}^{b}, \frac{C_{y}^{b}}{B_{y}^{b}}\right) \geq 1 \text { and } R_{z}\left(B_{y}^{b}, B_{z}^{b}, \frac{C_{z}^{b}}{B_{z}^{b}}\right) \geq 1
$$

the viability kernel associated with the dynamics $f$ in (1) and the acceptable set $\mathbb{D}$ in (26) is given by

$$
\mathbb{V}(f, \mathbb{D})=\left\{(y, z) \mid y \geq B_{y}^{b}, z \geq B_{z}^{b}, y R_{y}\left(y, z, \frac{C_{y}^{b}}{y}\right) \geq B_{y}^{b}, z R_{z}\left(y, z, \frac{C_{z}^{b}}{z}\right) \geq B_{z}^{b}\right\}
$$

Proof. According to induction (24), we have:

$$
\begin{aligned}
& \mathbb{V}_{0}=\left\{(y, z) \mid y \geq B_{y}^{b}, z \geq B_{z}^{b}\right\}, \\
& \mathbb{V}_{1}=\left\{(y, z) \mid \begin{array}{c}
y \geq B_{y}^{b}, z \geq B_{z}^{b} \text { and, for some }(v, w) \geq 0, \\
v y \geq C_{y}^{b}, w z \geq C_{z}^{b}, y R_{y}(y, z, v) \geq B_{y}^{b}, z R_{z}(y, z, w) \geq B_{z}^{b}
\end{array}\right\} \\
& =\left\{(y, z) \mid y \geq B_{y}^{b}, z \geq B_{z}^{b}, y R_{y}\left(y, z, \frac{C_{y}^{b}}{y}\right) \geq B_{y}^{b}, z R_{z}\left(y, z, \frac{C_{z}^{b}}{z}\right) \geq B_{z}^{b}\right\} \\
& \text { because } v \mapsto R_{y}(y, z, v) \text { and } w \mapsto R_{z}(y, z, w) \text { are decreasing, } \\
& \text { and thus we may select } v=\frac{C_{y}^{b}}{y}, w=\frac{C_{z}^{b}}{z} \text {. } \\
& \text { Denoting } y^{\prime}=y R_{y}(y, z, v), z^{\prime}=z R_{z}(y, z, w) \text {, we obtain, } \\
& \mathbb{V}_{2}=\left\{\begin{array}{l|l}
(y, z) & \begin{array}{l}
y \geq B_{y}^{b}, z \geq B_{z}^{b} \text { and, for some }(v, w) \geq 0, \\
v y \geq C_{y}^{b}, w z \geq C_{z}^{b} \\
y^{\prime} \geq B_{y}^{b}, y^{\prime} R_{y}\left(y^{\prime}, z^{\prime}, \frac{C_{y}^{b}}{y^{\prime}}\right) \geq B_{y}^{b}, z^{\prime} \geq B_{z}^{b}, z^{\prime} R_{z}\left(y^{\prime}, z^{\prime}, \frac{C_{z}^{b}}{z^{\prime}}\right) \geq B_{z}^{b}
\end{array}
\end{array}\right\} .
\end{aligned}
$$

We shall now make use of the property, recalled in Sect. A, that when the decreasing sequence $\left(\mathbb{V}_{k}\right)_{k \in \mathbb{N}}$ of viability kernels up to time $k$ is stationary, its limit is the viability kernel $\mathbb{V}(f, \mathbb{D})$. Hence, it suffices to show that $\mathbb{V}_{1} \subset \mathbb{V}_{2}$ to obtain that $\mathbb{V}(f, \mathbb{D})=\mathbb{V}_{1}$. Let $(y, z) \in \mathbb{V}_{1}$, so that

$$
y \geq B_{y}^{b}, \quad z \geq B_{z}^{b} \text { and } y R_{y}\left(y, z, \frac{C_{y}^{b}}{y}\right) \geq B_{y}^{b}, \quad z R_{z}\left(y, z, \frac{C_{z}^{b}}{z}\right) \geq B_{z}^{b} .
$$

Since $R_{y}: \mathbb{R}^{3} \rightarrow \mathbb{R}$ is continuously decreasing in the control variable, with $\lim _{v \rightarrow+\infty} R_{y}(y, z, v) \leq 0$, and since $y R_{y}\left(y, z, \frac{C_{y}^{b}}{y}\right) \geq B_{y}^{b}$, there exists a $\hat{v} \geq \frac{C_{y}^{b}}{y}$ (depending on $y$ and $z$ ) such that $y^{\prime}=y R_{y}(y, z, \hat{v})=B_{y}^{b}$. The same holds for $R_{z}: \mathbb{R}^{3} \rightarrow \mathbb{R}$ and $z^{\prime}=z R_{z}(y, z, \hat{w})=B_{z}^{b}$. By (27), we deduce that

$$
y^{\prime} R_{y}\left(y^{\prime}, z^{\prime}, \frac{C_{y}^{b}}{y^{\prime}}\right)=B_{y}^{b} R_{y}\left(B_{y}^{b}, B_{z}^{b}, \frac{C_{y}^{b}}{B_{y}^{b}}\right) \geq B_{y}^{b} \text { and } z^{\prime} R_{z}\left(y^{\prime}, z^{\prime}, \frac{C_{z}^{b}}{z^{\prime}}\right)=B_{z}^{b} R_{z}\left(B_{y}^{b}, B_{z}^{b}, \frac{C_{z}^{b}}{B_{z}^{b}}\right) \geq B_{z}^{b} .
$$

The inclusion $\mathbb{V}_{1} \subset \mathbb{V}_{2}$ follows. 
Corollary 6 Suppose that the assumptions of Proposition 2 are satisfied. Denoting

$$
\left\{\begin{array}{l}
\hat{v}(y, z)=\max \left\{v \geq \frac{C_{y}^{b}}{y} \mid y R_{y}(y, z, v)=y^{b}\right\} \\
\hat{w}(y, z)=\max \left\{w \geq \frac{C_{z}^{b}}{z} \mid z R_{z}(y, z, w)=z^{b}\right\}
\end{array}\right.
$$

the set of viable controls is given by

$$
\mathbb{U}_{\mathbb{V}(f, \mathbb{D})}(y, z)=\left\{\begin{array}{l|l}
(v, w) & \begin{array}{l}
\hat{v}(y, z) \geq v \geq \frac{C_{y}^{b}}{y}, \quad \hat{w}(y, z) \geq w \geq \frac{C_{z}^{b}}{z}, \\
y^{\prime} R_{y}\left(y^{\prime}, z^{\prime}, \frac{C_{y}^{b}}{y^{\prime}}\right) \geq y^{b}, z^{\prime} R_{z}\left(y^{\prime}, z^{\prime}, \frac{C_{z}^{b}}{z^{\prime}}\right) \geq z^{b}
\end{array}
\end{array}\right\},
$$

where $y^{\prime}=y R_{y}(y, z, v), z^{\prime}=z R_{z}(y, z, w)$.

\section{B.2 Proof of Proposition 4}

Proof. By (9), and the property that both $R_{y}$ and $R_{z}$ are decreasing in the control variable, the quantities (8) exist.

Also since both $R_{y}$ and $R_{z}$ are decreasing in the control variable, we obtain that

$$
R_{y}\left(B_{y}^{b}, B_{z}^{b}, \frac{C_{y}^{b, \star}\left(y_{0}, z_{0}\right)}{B_{y}^{b}}\right) \geq R_{y}\left(B_{y}^{b}, B_{z}^{b}, \frac{C_{y}^{b, \star}}{B_{y}^{b}}\right)=1 \text { and } R_{z}\left(B_{y}^{b}, B_{z}^{b}, \frac{C_{z}^{b, \star}\left(y_{0}, z_{0}\right)}{B_{z}^{b}}\right) \geq R_{z}\left(B_{y}^{b}, B_{z}^{b}, \frac{C_{z}^{b, \star}}{B_{z}^{b}}\right)=1 .
$$

To end up, the above inequalities and the assumption that $y_{0} \geq B_{y}^{b}$ and $z_{0} \geq B_{z}^{b}$ allow us to conclude, thanks to Proposition 5 , that $\left(y_{0}, z_{0}\right)$ belongs to the viability kernel $\mathbb{V}(f, \mathbb{D})$ given in $(28)$.

In other words, starting from the initial point $\left(y\left(t_{0}\right), z\left(t_{0}\right)\right)=\left(y_{0}, z_{0}\right)$, there exists an appropriate harvesting path which can provide, for all time, at least the catches (8).

\section{References}

Jürgen Alheit and Miguel Niquen. Regime shifts in the Humboldt Current Ecosystem. Progress in Oceanography, 60:201-222, 2004.

J-P. Aubin. Viability Theory. Birkhäuser, Boston, 1991. 542 pp.

Michael Ballón, Claudia Wosnitza-Mendo, Renato Guevara-Carrasco, and Arnaud Bertrand. The impact of overfishing and El Niño on the condition factor and reproductive success of Peruvian hake, Merluccius gayi peruanus. Progress In Oceanography, 79(2-4):300 - 307, 2008.

C. Béné and L. Doyen. Sustainability of fisheries through marine reserves: a robust modeling analysis. Journal of Environmental Management, 69(1):1-13, 2003. 
C. Béné, L. Doyen, and D. Gabay. A viability analysis for a bio-economic model. Ecological Economics, 36:385-396, 2001.

Arnaud Bertrand, Marceliano Segura, Mariano Gutiérrez, and Luis Vásquez. From small-scale habitat loopholes to decadal cycles: a habitat-based hypothesis explaining fluctuation in pelagic fish populations off Peru. Fish and Fisheries, 5:296-316, 2004.

D. Bertsekas and I. Rhodes. On the minimax reachability of target sets and target tubes. Automatica, 7:233-247, 1971.

Laetitia Chapel, Guillaume Deffuant, Sophie Martin, and Christian Mullon. Defining yield policies in a viability approach. Ecological Modelling, 212(1-2):10 - 15, 2008.

David M. Checkley, Jürgen Alheit, Yoshioki Ooseki, and Claude Roy. Climate Change and Small Pelagic Fish. Cambridge University Press, 2009.

C. W. Clark. Mathematical Bioeconomics. Wiley, New York, second edition, 1990.

F. H. Clarke, Y. S. Ledayev, R. J. Stern, and P. R. Wolenski. Qualitative properties of trajectories of control systems: a survey. Journal of Dynamical Control Systems, 1:1-48, 1995.

M. De Lara and L. Doyen. Sustainable Management of Natural Resources. Mathematical Models and Methods. Springer-Verlag, Berlin, 2008.

M. De Lara and V. Martinet. Multi-criteria dynamic decision under uncertainty: A stochastic viability analysis and an application to sustainable fishery management. Mathematical Biosciences, 217(2):118-124, February 2009.

M. De Lara, L. Doyen, T. Guilbaud, and M.-J. Rochet. Is a management framework based on spawning-stock biomass indicators sustainable? A viability approach. ICES J. Mar. Sci., 64(4): 761-767, 2007.

L. Doyen and M. De Lara. Stochastic viability and dynamic programming. Systems and Control Letters, 59(10):629-634, October 2010.

K. Eisenack, J. Sheffran, and J. Kropp. The viability analysis of management frameworks for fisheries. Environmental Modeling and Assessment, 11(1):69-79, February 2006. 
FAO. Indicators for sustainable development of marine capture fisheries. FAO Technical Guidelines for Responsible Fisheries 8, FAO, 1999. 68 pp.

S. Garcia, A. Zerbi, C. Aliaume, T. Do Chi, and G. Lasserre. The ecosystem approach to fisheries. Issues, terminology, principles, institutional foundations, implementation and outlook. FAO Fisheries Technical Paper, 443(71), 2003.

C. Hall. An assessment of several of the historically most influential theoretical models used in ecology and of the data provided in their support. Ecological Modelling, 43(1-2):5-31, 1988.

Anne B. Hollowed, Nicholas Bax, Richard Beamish, Jeremy Collie, Michael Fogarty, Patricia Livingston, John Pope, and Jake C. Rice. Are multispecies models an improvement on single-species models for measuring fishing impacts on marine ecosystems? ICES J. Mar. Sci., 57(3):707-719, 2000.

ICES. Report of the ICES advisory committee on fishery management and advisory committee on ecosystems, 2004. ICES Advice, 1, ICES, 2004. 1544 pp.

IMARPE. Trabajos expuestos en el taller internacional sobre la anchoveta peruana (TIAP), 9-12 Mayo 2000. Bol. Inst. Mar Peru, 19:1-2, 2000.

IMARPE. Report of the first session of the international panel of experts for assessment of Peruvian hake population. March 2003. Bol. Inst. Mar Peru, 21:33-78, 2004.

Stephen L. Katz, Richard Zabel, Chris Harvey, Thomas Good, and Phillip Levin. Ecologically sustainable yield. American Scientist, 91(2):150, March-April 2003.

P. A. Larkin. An Epitaph for the Concept of Maximum Sustained Yield. Transactions of the American Fisheries Society, 106(1):1-11, January 1977.

C. Mullon, P. Cury, and L. Shannon. Viability model of trophic interactions in marine ecosystems. Natural Resource Modeling, 17:27-58, 2004.

J. D. Murray. Mathematical Biology. Springer-Verlag, Berlin, third edition, 2002.

Daniel Pauly and María Lourdes Palomares. New estimates of monthly biomass, recruitment and related statistics of anchoveta (Engraulis ringens) off Peru (4-14º' S), 1953-1985, pages 189-206. 18. ICLARM Conference Proceedings, 1989. 
PRODUCE. Establecen régimen provisional de pesca del recurso merluza correspondiente al año 2006. El Peruano, page 307804, 30 de diciembre 2005. RM-356-2005-PRODUCE.

PRODUCE. Establecen régimen provisional de pesca del recurso merluza correspondiente al año 2007. El Peruano, page 335485, 27 de diciembre 2006. RM-357-2006-PRODUCE.

A. Rapaport, J.-P. Terreaux, and L. Doyen. Sustainable management of renewable resource: a viability approach. Mathematics and Computer Modeling, 43(5-6):466-484, March 2006.

P. Saint-Pierre. Approximation of viability kernel. Applied Mathematics and Optimization, 29: 187-209, 1994.

M. B. Schaefer. Some aspects of the dynamics of populations important to the management of commercial marine fisheries. Bulletin of the Inter-American tropical tuna commission, 1:25-56, 1954.

Chin-Hwa Sun, Fu-Sung Chiang, Te-Shi Liu, and Ching-Cheng Chang. A welfare analysis of El Niño forecasts in the international trade of fish meal - an application of stochastic spatial equilibrium model. 2001 Annual meeting, August 5-8, Chicago, IL 20770, American Agricultural Economics Association (New Name 2008: Agricultural and Applied Economics Association), 2001.

J. Tam, S. Purca, L. O. Duarte, V. Blaskovic, and P. Espinoza. Changes in the diet of hake associated with El Niño 1997-1998 in the Northern Humboldt Current ecosystem. Advances in Geosciences., 6:63-67, 2006. 